EESTI NSV TEADUSTE AKADEEMIA TOIMETISẼD. 29. KOIDË FUOSIKA * MATEMAATIKA. 1980, NR. 4

ИЗВЕСТИЯ АКАДЕМИИ НАУК ЭСТОНСКОИ ССР. ТОМ 29 ФИЗИКА * МАТЕМАТИКА. 1980, № 4

\title{
НЕСТАЦИОНАРНОЕ РАССЕЯНИЕ АКУСТИЧЕСКИХ ВОЛН НА УПРУГОМ ЦИЛИНДРЕ С УПРУГИМ ЗАПОЛНИТЕЛЕМ
}

\author{
(Представил Н. Алумяэ)
}

Описываются результаты расчета частотной и временной зависимостей акустического поля давления, рассеянного цилиндром, проводится их детальный анализ и исследуются информативные признаки. Во временной зависимости выделяются основные компоненты. Дается сопоставление результатов с рассчитанными для предельных случаев. Предлагается способ решения инверсной задачи.

Проблема рассеяния акустического волнового пакета на упругих, сплошных либо полых, цилиндрах и сферах исследовалась многими авторами. Библиография по этому вопросу охватывает сотни статей, часть из которых отражена в монографиях и обзорах $\left[{ }^{1-5}\right]$. Работ, посвященных проблеме рассеяния на многослойном упругом препятствии, значительно меньше $\left[{ }^{6-8}\right]$. Две пионерские работы в этой области $\left[{ }^{7,8}\right]$ исследуют рассеяние волн на заполненном жидкостью толстостенном упругом цилиндре, покрытом вязкоупругим поглощающим слоем. При малых волновых размерах решение представляется в виде ряда по собственным функциям и доводится до частотной зависимости, а при больших размерах к ряду применяется преобразование Зоммерфельда-Ватсона, что позволяет рассчитать полярные диаграммы направленности.

Полученные к настоящему времени решения поставляют частотную зависимость, что дает возможность судить о происходящих явлениях лишь для установившихся режимов. В данной статье приводятся как частотная, так и временная зависимости, повышающие информативность ответа и допускающие адекватную физическую интерпретацию.

\section{1. Постановка задачи, метод решения, точность вычисления}

Рассматривается следующая задача математической физики. В безграничной акустической среде находится толстостенный упругий круговой цилиндр бесконечной протяженности, внутри которого содержится коаксиальный сплошной упругий заполнитель. На цилиндр набегает волновой пакет с плоским фронтом, параллельным продольной оси цилиндра. Требуется рассчитать нестационарное двумерное поле давления в акустической среде, вызванное рассеянием волнового пакета на цилиндре.

Математически задача сводится к решению пяти волновых уравнений, одно из которых описывает движение акустической среды, а остальные - движение упругого цилиндра и его заполнителя (по два уравнения для каждого), связанных между собой посредством семи условий контакта на двух цилиндрических границах раздела. На границе раз- 
дела акустичесқой и упругой сред имеют меето три условия контакта: непрерывность радиальных перемещений и напряжений, отсутствие тангенциальных напряжений. На границе раздела двух упругих сред выполняются четыре условия: непрерывность радиальных и тангенциальных перемещений и напряжений. Контакт на этой границе является полным: сплошной заполнитель «впаян» в толстостенный цилиндр. Полагается, что все неизвестные функции ограничены в областях, где они определены. Принимается, что процесс рассеяния начинается в момент, когда падающий волновой пакет коснется цилиндра. До этого все искомые функции и их первые производные по времени считаются равными нулю.

При решении задачи выпишем уравнения движения в цилиндрической системе координат, продольная ось которой совпадает с осью симметрии цилиндра. Применим интегральное преобразование Фурье по времени ( $F$-преобразование). В пространстве $F$-преобразования волновые уравнения переходят в уравнения Гельмгольца. Используем метод разделения переменных по радиальной и угловой координатам; решим появляющиеся при этом обыкновенные дифференциальные уравнения и для каждой из искомых функций запишем точное аналитическое решение в виде бесконечного ряда, коэффициенты которого находятся из решения системы семи алгебраических уравнений (для каждого значения индекса суммирования), получающейся при удовлетворении условий контакта в пространстве $F$-преобразования. Обратное преобразование Фурье выполним численно. Подробно метод решения и алгоритм вычисления излагаются в $\left[{ }^{2}\right]$.

Здесь отметим некоторые детали. Частотная зависимость (спектральная плотность) считалась с шагом $\Delta x=10 / 256$ в интервале частот $x=0-100$, а временная зависимость - с шагом $\Delta \tau=0,005$ в интервале $\tau=0$ - 16. При численном интегрировании использовалась экономная процедура Ричардсона-Ромберга. Зафиксировав частоту и длительность падающего волнового пакета, мы подбирали шаг и интервал интегрирования таким образом, чтобы результаты расчета при уменьшении шага вдвое (либо при увеличении интервала вдвое) мало различались между собой. Вычисления проводились на ЭВМ ЕС-1033. Суммарная погрешность наших вычислений не превышает половины процента.

Будем применять обозначения, введенные в $\left[{ }^{9}\right]$, снабдив упругие постоянные для заполнителя индексом нуль.

Используем безразмерные переменные

$$
r=R / a, \quad t=c_{0} T / a .
$$

Зависимость изменения давления в падающем волновом пакете может быть задана произвольно; нами она выбрана синусоидальной

$$
p_{i}=p_{*} \sin x_{0} \delta\left[H(\delta)-H\left(\delta-\delta_{0}\right)\right], \quad \delta=t+(r \cos \theta-1),
$$

где $p$. - постоянная, имеющая размерность давления; $x_{0}-$ частота заполнения; $\delta_{0}$ - длительңость волнового пакета; $H(\delta)-$ единичная функция Хевисайда. Период частоты заполнения обозначим через $\tau_{0}=2 \pi / x_{0}$.

Отметим, что при введенных безразмерных переменных наружный радиус цилиндра равен единице; за единицу времени плоский фронт падающего волнового пакета проходит расстояние, равное единице; частота заполнения является одновременно и волновым числом в акустической среде. 
Нормируем давление в рассеянном поле на геометрический множитель

$$
p=p_{s} / m, \quad m=p_{*}[r(2 \cos \gamma / \cos \alpha-1 / r)]^{-1 / 2} .
$$

При заданном угле наблюдения $\theta$ (либо при известном угле падения $\alpha$ ) между углами имеют место следующие соотношения

$$
\sin \alpha=r \sin \gamma, \quad \theta+\gamma-2 \alpha=0 .
$$

Давление в некоторой фиксированной точке наблюдения в акустической среде $p(\tau)$ будем исследовать с момента, когда рассеянное упругим цилиндром поле придет в эту точку: $\tau=t-(1-2 \cos \alpha+r \cos \gamma)$. Задание «сдвинутого» времени $\tau$ проводилось с позиций лучевого метода и полагалось, что точка наблюдения находится в области зеркального отражения.

Обозначим $F$-преобразование давления $p(\tau)$ через $p^{F}(x)$. Для краткости будем называть $p(\tau)$ временной зависимостью, а $\left|p^{F}(x)\right|$ - частотной зависимостью.

Расчеты проводились для случая размещения в воде стального цилиндра, заполненного плавленным кремнием, в точке наблюдения с координатами $r=10^{4}$ и $\theta=0$ при следующих параметрах

$$
\begin{aligned}
& \mathrm{Q}_{0}=1,00 \mathrm{r} / \mathrm{Cm}^{3}, \quad c_{0}=1493 \mathrm{~m} / \mathrm{c}, \\
& \mathrm{Q}_{1}=7,70 \mathrm{r} / \mathrm{cm}^{3}, \quad c_{1}=5960 \mathrm{M} / \mathrm{c}, \quad c_{2}=3240 \mathrm{M} / \mathrm{c}, \\
& \mathrm{Q}_{10}=2,20 \mathrm{z} / \mathrm{CM}^{3}, \quad c_{10}=5968 \mathrm{M} / \mathrm{c}, \quad c_{20}=3764 \mathrm{M} / \mathrm{c}, \\
& \beta=b / a=0,68138 \text {. }
\end{aligned}
$$

Отметим, что метод, алгоритм и программа вычислений не накладывают ограничений на координаты точки наблюдения.

\section{2. Частотная зависимость}

На рис. 1 показана частотная зависимость - модуль функции $p^{F}(x)$. В интервале частот $x=0-30$ кривая имеет весьма регулярный характер, поскольку здесь преобладает одна мода. Начиная с $x>30$ регулярность нарушается в связи с тем, что включается и следующая мода. В дальнейшем по мере роста частоты растет число мод, изрезаннее становится кривая и падает величина ее размаха.

С целью анализа положения и величины экстремумов частотной зависимости в случае рассеяния на упругом цилиндре с упругим заполнителем (в дальнейшем называемом препятствием $E E$ ) мы провели сопоставление частотных зависимостей препятствия $E E$ и его предельных случаев: «акустически жесткого» цилиндра $(R)$; жидкого цилиндра с коаксиальным жидким заполнителем $(F F)$, предположив, что параметры Ляме материалов упругого цилиндра и его заполнителя $\mu$ и $\mu_{0}$ равны нулю, а остальные параметры сохранены; толстостенного упругого цилиндра без заполнителя $(S E)$. В интервале частот $x=0-20$ результаты показаны на рис. 2.

Для каждой нз модельных задач кривая частотной зависимости имеет достаточно простой и регулярный характер. В интервале $x=$ $=0-4$ поведение кривых сходно, за исключением кривой $S E$. В интервале частот $x=4-20$ экстремумы кривых $E E$ и $S E$ в целом повторяют друг друга, хотя максимумы и минимумы кривой $E E$ следуют с некоторым регулярным отставанием (тем бо́льшим, чем выше частота), а 
Нестащионарное рассеяние акустических волн...
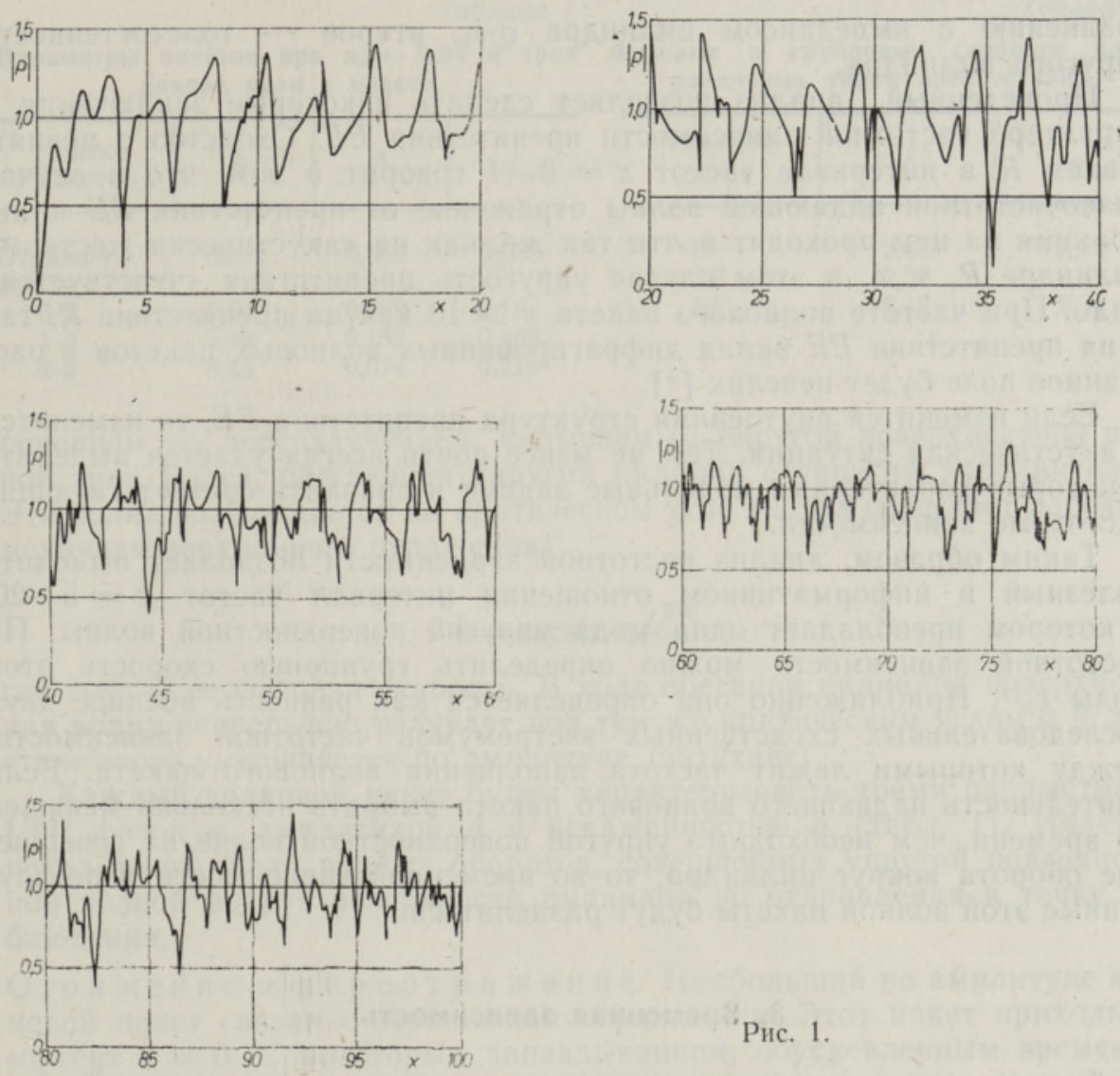

'Рис. 1.

их пики срезаны и закруглены. Это соответствие удается проследить до тех пор, пока разность между координатами $x$ двух последовательных максимумов кривой $S E$ не становится меньше разности координат двух сходственных максимумов кривых $E E$ и $S E$. Характерно, что в интервале частот $x=4-20$ кривая $E E$ «передает» минимумы предельного объекта $F F$.

C точки зрения собственных форм колебаний упругой системы, окруженной акустической средой, эти результаты можно интерпретировать следующим образом. В интервале частот $x=0-4$ в препятствии $E E$ возбуждаются формы колебаний, сходные по форме с колебаниями препятствия $R$ (т. е. в упруго-жидкой системе преобладают колебания, характерные для окружающей цилиндр жидкости), в интервале $x=$ $=4-20-$ близкие по форме колебаниям препятствия $S E$, а при умеренных частотах $x>20$ - уже отличные от форм колебаний предельных объектов.

Отметим, что у препятствия $E E$ нет «чистых» форм колебаний, как у предельных объектов $R, F F, S E$. В некоторых интервалах частот у препятствия $E E$ преобладают формы колебаний, близкие к «чистым»; последние наступают лишь тогда, когда выполняется предельный переход (как относительно уравнений движения, так и относительно условий контакта). Здесь легко видеть, что близость форм колебаний препятствий $E E$ и $S E$ в интервале частот $x=4-20$ обусловливают два обстоятельства: первое - малость импеданса заполнителя @10 $c_{10}$ по 
сравнению с импедансом цилиндра @ $c_{1}$, второе - толстостенность упругого цилиндра.

Проведенный анализ позволяет сделать некоторые заключения о характере частотной зависимости препятствия $E E$. Сходство с препятствием $R$ в интервале частот $x=0-4$ говорит о том, что в случае низкочастотной падающей волны отражение от препятствия $E E$ и дифракция на нем проходят почти так же, как на «акустически жестком» цилиндре $R$, т. е. в этом случае упругость препятствия «чувствуется» мало. При частоте волнового пакета $x>10$ как на препятствии $R$, так и на препятствии $E E$ вклад дифрагированных волновых пакетов в рассеянное поле будет невелик $\left[{ }^{9}\right]$.

Если изменится внутренняя структура препятствия $E E$, то изменится и акустическая ситуация. Тем не менее почти всегда удается выделить некоторые характерные модельные задачи и сравнить соответствующие частотные зависимости.

Таким образом, анализ частотной зависимости позволяет выделить полезный в информативном отношении интервал частот $x=5-20$, в котором преобладает одна мода упругой поверхностной волны. По частотной зависимости можно определить групповую скорость этой моды $c_{3}{ }^{g r}$. Приближенно она определяется как разность аб́сцисс двух последовательных сходственных экстремумов частотной зависимости, между которыми лежит частота заполнения волнового пакета. Если длительность падающего волнового пакета выбрать несколько меньшей по времени, чем необходимо упругой поверхностной волне. на совершение оборота вокруг цилиндра, то во временной зависимости переизлученные этой волной пакеты будут разделяться.

\section{3. Временная зависимость}

Выберем частоту заполнения волнового пакета в информативной области частотной зависимости $x_{0}=9,84$ и длительность волнового пакета $\delta_{0}=3 \tau_{0}$. В рассматриваемой акустической ситуации длина волны со-

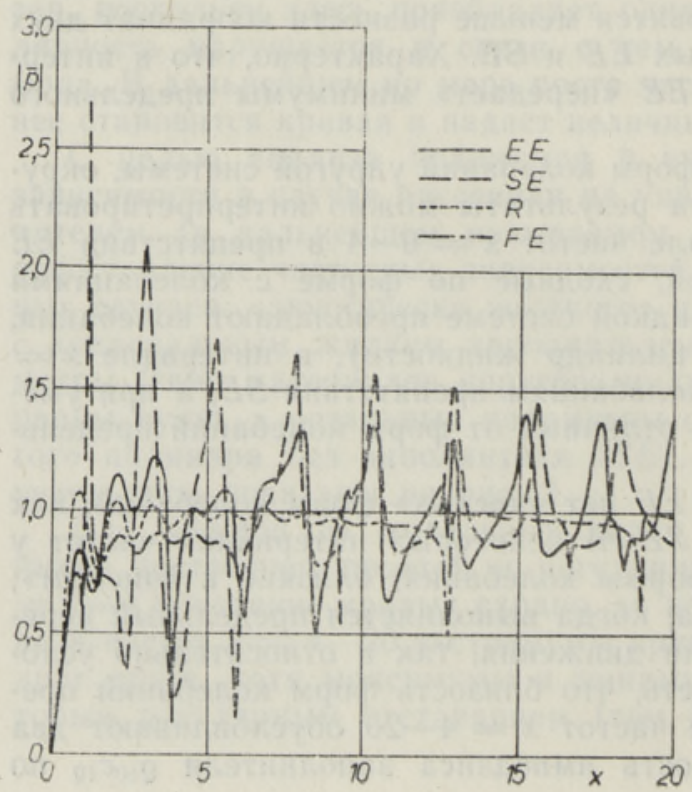
ставляет 0,64 , а длина волнового пакета - 1,92. Выполняя обратное преобразование Фурье, проведем численное интегрирование в интервале частот $x=$ $=0-100$. В результате для препятствия $E E$ получим временную зависимость, изображенную на рис. 3.

Один единственный падающий волновой пакет вызывает серию пакетов во временной зависимости. Первый из них, в дальнейшем называемый отраженным, обусловлен процессами отражения и переотражения, а последующие, в

Рис. 2. 
Параметры пакетов при $x_{0}=9,84$ и трех длинах волн в пакете

\begin{tabular}{l|c|c|c}
\hline $\begin{array}{c}\text { Индекс } \\
\text { пакета }\end{array}$ & $\varphi$ & $A$ & $d$ \\
\hline $\begin{array}{l}\text { Отражен- } \\
\text { ный }\end{array}$ & 0,012 & 0,964 & 0,089 \\
0-й & 2,50 & 0,272 & 0,185 \\
1-й & 5,49 & 0,152 & 0,466 \\
2 -й & 8,45 & 0,074 & 0,216
\end{tabular}

Фазовые и групповые скорости для различных типов препятствий

\begin{tabular}{l|l|l}
\hline $\begin{array}{c}\text { Препят- } \\
\text { ствне }\end{array}$ & $c_{3}{ }^{\text {ph }}$ & $c_{3^{g r}}$ \\
\hline & & \\
$E$ & 2,42 & 2,42 \\
$S E$ & 2,58 & 2,58 \\
$E E$ & 2,12 & 2,34
\end{tabular}

основном, - переизлучением, вызываемым упругой поверхностной волной типа Рэлея, обегающей упругий цилиндр с упругим заполнителем. Эта волна возбуждается на критическом угле (когда перестает работать механизм зеркального отражения)

$$
\theta=\arcsin c_{0} / c_{3}^{p h}
$$

где $c_{3}{ }^{p h}$ - ее фазовая скорость. В ходе обегания цилиндра поверхностная волна непрерывно излучает под тем же критическим углом и вследствне этого уменьшается по амплитуде (затухает).

Каждый волновой пакет будем характеризовать тремя параметрами $\left[{ }^{10}\right]$ : фазой $\varphi$, амплитудой $A$ и искаженностью $d$ (табл. 1). Индексы показывают число полных оборотов, совершенных упругой поверхностной волной вокруг поверхности цилиндра до отправления в точку наблюдения.

Отражение и пе ре о т а жен ие. Наибольший по амплитуде волновой пакет связан с зеркальным отражением. Этот пакет приходит в момент $\tau=0$. С некоторым запаздыванием, обусловленным временем, необходимым на пробегание толщины упругого цилиндра, на него накладывается волновой пакет, отраженный от поверхности $r=\beta$. Если $3 \tau_{0}>2 h c_{0} / c_{1}$ ( $h=H / a$ - безразмерная толщина упругого цилиндра), то на конец первого переотраженного волнового пакета накладывается начало второго переотраженного и т. д. При достаточной длительности падающего волнового пакета на отраженный будут накладываться па-

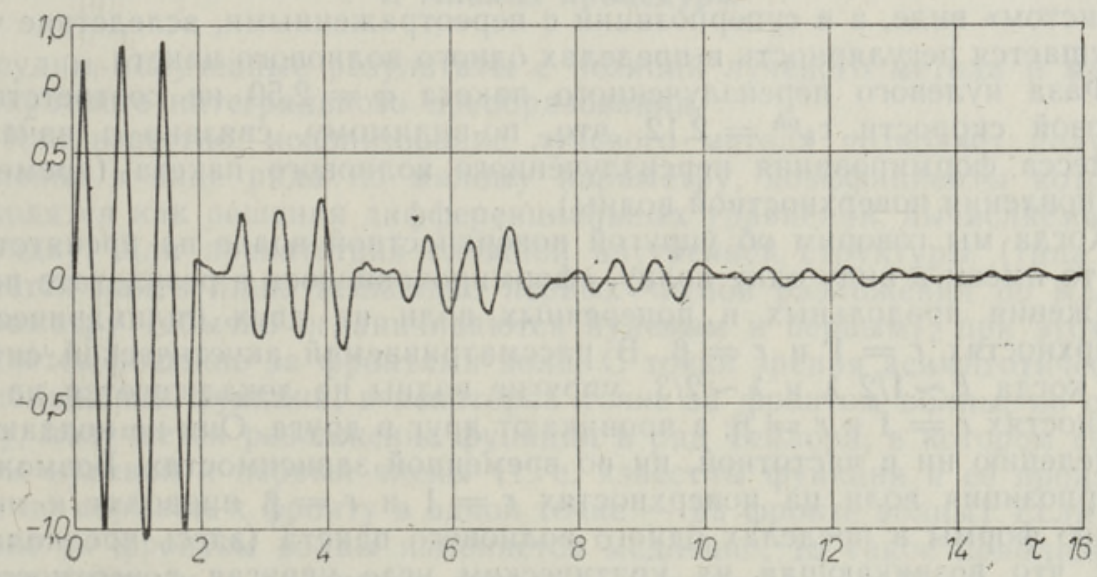

Рис. 3 , 


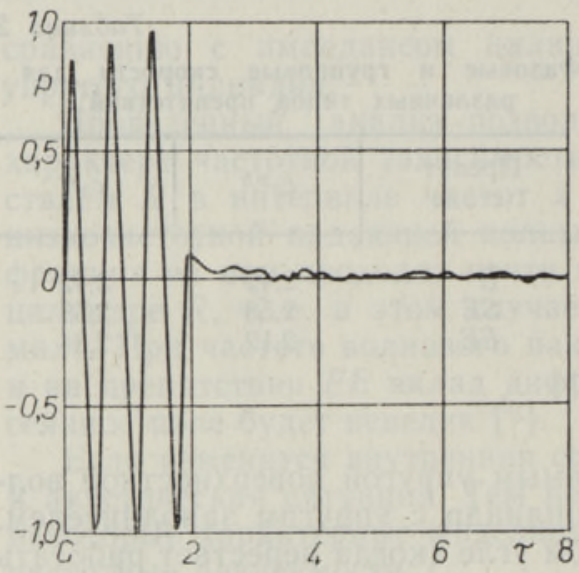

Рис. 4.

кеты, переотраженные от задней поверхности заполнителя и от задней поверхности цилиндра. Поэтому первый пакет, ради краткости называемый отраженным, может принять достаточно сложную форму из-за суперпозиции многочисленных волновых пакетов, различных по времени прихода, фазе и амплитуде.

Как известно, на плоской границе раздела акустической и упругой сред в случае нормального падения волны упругая среда ведет себя как акустическая - на поверхности раздела поперечные волны не возникают. На цилиндрической границе раздела подобное имеет место на нулевом угле падения. На отличных от нуля углах падения возникают поперечные волны, амплитуды которых невелики вследствие непрерывности по углу. Из-за появления поперечных волн меняется и коэффициент отражения (по сравнению с коэффициентом при нулевом угле падения). Чтобы оценить эффект появления поперечных волн, мы провели расчет временной зависимости для препятствия $F F$, где поперечные волны не возникают. Оказалось, что положение и величина максимумов и минимумов временной зависимости для препятствий $E E$ и $F F$ (ср. рис. 3 и 4) практически совпадают в интервале $\tau=0-1,92$, занимаемом отраженным пакетом. Это свидетельствует о том, что эффект появления поперечных волн и их вклад в рассеянное препятствием $E E$ поле невелики при нулевом угле наблюдения. Длинный «хвост» временной зависимости в случае препятствия $F F$ обусловлен процессом переотражения; его амплитуда мала. Подобный «хвост» имеет место и в случае препятствия $E E$.

П ер е и з лучен и е. Характерной особенностью временной зависимости в случае рассеяния на упругом препятствии является наличие переизлученных волновых пакетов (ср. рис. 3 и 4). Эти пакеты появляются не в «чистом» виде, а в суперпозиции с переотраженными, вследствие чего нарушается регулярность в пределах одного волнового пакета.

Фаза нулевого переизлученного пакета $\varphi=2,50$ не соответствует фазовой скорости $c_{3}{ }^{p h}=2,12$, что, по-видимому, связано с началом процесса формирования переизлученного волнового пакета (временем установления поверхностной волны).

Когда мы говорим об упругой поверхностной волне на препятствии $E E$, то имеем в виду одну волну, сформировавшуюся в результате переотражения продольных и поперечных волн на двух цилиндрических поверхностях $r=1$ и $r=\beta$. В рассматриваемой акустической ситуации, когда $h \sim 1 / 2 \lambda$ и $\lambda \sim 2 / 3$, упругие волны не локализуются на поверхностях $r=1$ и $r=\beta$, а проникают друг в друга. Они не поддаются разделению ни в частотной, ни во временной зависимостях. Возможно, суперпозиция волн на поверхностях $r=1$ и $r=\beta$ приводит к искажению формы в пределах одного волнового пакета (здесь предполагается, что возникающая на критическом угле упругая поверхностная волна имеет постоянную в пределах одного пакета амплитуду). 


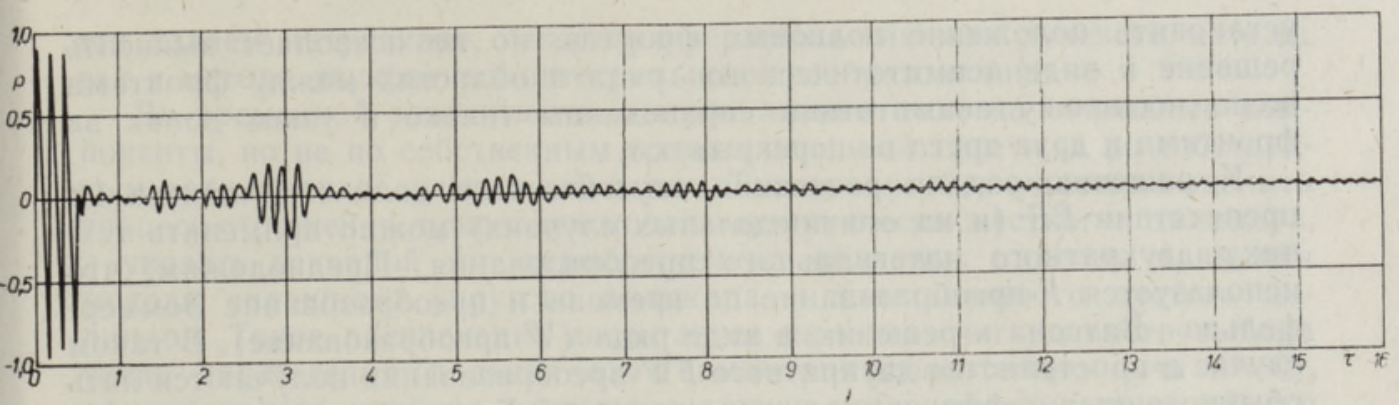

Рис. 5.

Мы сопоставили амплитуды нулевых переизлученных волновых пакетов для трех различных типов препятствий: $E$ - сплошного упругого цилиндра, $S E$ и $E E$ (для препятствий $E$ и $S E$ упругие параметры соответствовали параметрам наружного цилиндра). Оказалось, что эти амплитуды практически совпадают. Значит, доля энергии, вносимой в упругую преграду, одинакова для указанных типов препятствий, что представляется естественным, поскольку условия на границе раздела $r=1$ у них одинаковы. Различие во внутренней структуре наиболее ярко проявляется в различии фазовых и групповых скоростей (см. табл. 2).

Ясно, что на препятствиях $E$ и $S E$ упругая поверхностная волна распространяется без дисперсии, а на препятствии $E E-$ с дисперсией. Впрочем, это заметно и по частотной зависимости.

Если акустическая ситуация изменится, то структура временной зависимости может стать совсем иной. В частности, при большой частоте заполнения и малой длительности падающего волнового пакета можно добиться отчетливого разделения переотраженных и переизлученных волновых пакетов. Такое разделение демонстрирует рис. 5. Временная зависимость была рассчитана при прежних параметрах препятствия $E E$ и новой частоте заполнения $x_{0}=39$. Получены следующие скорости распространения: $c_{3}{ }^{p h}=2,47 ; c_{3}{ }^{g r}=2,34$.

\section{4. Анализ процедуры}

Обсудим полученные результаты с позиций лучевого метода и метода двукратного интегрального преобразования.

Как известно, использование лучевого метода позволяет получить решение в виде ряда по малому параметру, коэффициенты которого находятся как решения дифференциальных уравнений, вычисляемых по порядку. Для препятствия сложной внутренней структуры (типа $E E$ ) удается найти лишь несколько первых членов разложения по малому параметру (обычно ограничиваются нулевым и первым); при этом получается решение за фронтами волн. С точки зрения асимптотического приближения функции, в некоторой точке за фронтом волны, по существу, получается разложение функции в ряд Тейлора, в котором сохранены нулевой и первый члены (т. е. известны функция и ее производная по нормали к фронту в одной точке - на фронте волны). Если давление за фронтом волны изменяется медленно, то такое приближение будет удачным, в противном случае - неадекватным. Лучевое приближение позволяет достаточно хорошо выделить отдельные типы волн и 
установить положение волновых фронтов, но не позволяет выписать решение в виде асимптотического ряда в областях между фронтами волң, поскольку асимптотики справедливы только в узких зонах за фронтами и друг друга не перекрывают.

K решению задачи рассеяния акустическо́го волнового пакета на препятствии $E E$ (и на его предельных случаях) можно применить технику двукратного интегрального преобразования. Предположим, что используется $F$-преобразование по времени и преобразование Зоммерфельда-Ватсона к решению в виде ряда (W-преобразование). В таком случае в пространстве двукратного $F W$-преобразования получаются пять обыкновенных дифференциальных уравнений Бесселя, связанных между собой посредством семи $F W$-преобразованных условий контакта. Легко выписать решения этих уравнений и найти постоянные интегрирования. В пространстве $F W$-преобразования можно разбить подынтегральную функцию на два слагаемых, одно из которых содержит полюса, а другое - нет. Последнее перегруппировкой можно преобразовать в бесконечную сумму, каждый член которой имеет особенность - седловую точку. Изменяя контур интегрирования, можно добиться того, чтобы он прошел через седловую точку. Вычисляя интеграл внутреннего обратного $W$-преобразования методом перевала, в принципе, можно получить бесконечную сумму $F$-преобразованных переотраженных волновых пакетов. Отметим, что в случае препятствия $E E$ на двух цилиндрических границах раздела имеется 25 индивидуальных коэффициентов отражения и прохождения, вообще говоря, зависящих от частоты. Вследствие повторных переотражений число волновых пакетов растет чрезвычайно быстро, а их амплитуды после каждого переотражения уменьшаются. Уследить за каждым переотраженным волновым пакетом, считая для него эйконал, фазу и амплитуду, трудно даже при небольшом числе переотражений. Характерно и то, что метод перевала в силу своей асимптотической природы описывает только зафронтовую область (либо область высоких частот). Так что за фронтами отдельных переотраженных волновых пакетов остаются зоны, для которых решение не найдено.

Первое слагаемое подынтегральной функции содержит полюса, которые часто объединяются в группы: полюса волн типа Франца, типа Стонели, типа Рэлея, типа шепчущей галереи (различные для продольных и поперечных волн). До выполнения внутреннего обратного $W$-преобразования надо вычислить координаты полюсов для всех групп. При аналитическом отыскании используются асимптотические разложения цилиндрических функций комплексного индекса, вследствие чего координаты полюсов на комплексной плоскости обычно удается найти только для больших частот. Численная процедура их отыскания достаточно трудоемка и тонка. При выполнении обратного $W$-преобразования следует найти вычеты в полюсах, причем эту процедуру приходится проводить с мелким шагом при большом интервале частоты. После выполнения обратного $F$-преобразования можно получить акустическое поле давления в виде суммы бегущих волн, но без выделения в ней основных компонентов. Хотя, в принципе, известно, как получить решение в виде суммы бегущих волн, временная зависимость этим методом не рассчитана даже для более простой задачи - о рассеянии на сплошном упругом цилиндре.

Использованный нами метод решения раскладывает рассеянное препятствием $E E$ акустическое поле давления в ряд по стоячим волнам. Фактически мы не можем указать буквально ни отраженный, ни переотраженные, ни переизлученные волновые пакеты. Тем не менее мы 
это делаем интуитивно, используя терминологию как лучевого метода, так и метода двукратных интегральных преобразований.

Во временной зависимости мы расчленяем поле на отдельные компоненты, но не по собственным волнам основной задачи, а по собственным волнам предельных к ней задач. Таким способом удается выделить процесс отражения, используя модель «акустически жесткого» цилиндра, и указать основной тип переизлученных волновых пакетов, применяя модели сплошного упругого цилиндра и толстостенного пустого цилиндра. Такое расчленение достигается нами за счет удачного выбора точки наблюдения (дальнее поле и симметрия относительно луча $\theta=0$ ), рационального подбора частоты и длительности падающего волнового пакета, большой разнице в импедансах сред, контактирующих на цилиндрических поверхностях $r=1$ и $r=\beta$. В результате удается выделить во временной зависимости основные и второстепенные компоненты.

\section{5. Инверсная задача}

Воспользуемся выявленными нами свойствами частотной и временной зависимостей поля давления, рассеянного препятствием $E E$, для решения инверсной задачи. По аналогии с прямой задачей будем считать, что на препятствие $E E$ падает акустический волновой пакет с плоским фронтом, параллельным продольной оси цилиндра. Для точки на луче $\theta=0$ в той части временной зависимости, которая определяется исключительно процессом отражения от наружной поверхности, давление имеет вид

$$
\begin{gathered}
p_{s}=p \cdot v[2(L+a) / a]^{-1 / 2} f(\tau), \\
v=(1-\zeta) /(1+\zeta), \quad \zeta=\varrho_{0} c_{0} / \varrho_{1} c_{1},
\end{gathered}
$$

где $v$ - коэффициент отражения от плоской границы раздела двух акустических сред при нормальном падении, $L$ - дальность от источника (он же и приемник) до наружной поверхности цилиндра. Здесь $f(\tau)$ рассчитана для случая «акустически жесткого» цилиндра того же радиуса $a$. Нам не требуется знания этой зависимости, поскольку мы будем использовать ее при одном и том же значении $\tau$. Дальность $L$ определяется по задержке отраженного волнового пакета по сравнению с падающим. Полагая известными значения давлений $p_{s 1}$ и $p_{s 2}$, вычисленных или зарегистрированных при одном и том же значении $\tau$, но двух различных дальностях, вычислим радиус цилиндра

$$
a=2\left(\alpha^{2} L_{1}-L_{2}\right) /\left(1-\alpha^{2}\right), \quad \alpha=p_{s 1} / p_{s 2} .
$$

Подставив его значение в формулу (7), найдем $v$ и $\varrho_{1} c_{1}$ :

$$
v=p_{s 1}\left[p_{*} f(\tau)\right]^{-1}\left[2\left(L_{1} f a\right) / a\right]^{1 / 2}, \quad \varrho_{1} c_{1}=\varrho_{0} c_{0}(1+v) /(1-v) .
$$

По таблице соответствий импеданс-материал $\left[{ }^{10}\right]$ определим физические параметры материала упругого цилиндра $\mathrm{Q}_{1}, c_{1}, c_{2}$.

Толщина $H$ определяется по задержке волнового пакета, переотраженного от границы раздела $r=\beta$, относительно пакета, отраженного от границы раздела $r=1$. Для отчетливого выделения начала прихода этого пакета можно вычесть из временной зависимости препятствия $E E$ временную зависимость препятствия $R$, умноженную на коэффнциент отражения $v$. Фаза переотраженного волнового пакета используется 
для определения толщины $H$, а его амплитуда - для определения импеданса заполнителя $0_{10} c_{10}$. Еще раз используя таблицу соответствий импеданс-материал, найдем параметры материала заполнителя @10, $c_{10}, c_{20}$.

Для проверки результатов решения инверсной задачи можно использовать их в качестве входных при решении прямой задачи и сравнить выходные данные с имеющимися.

\section{ЛИТЕРА Т Р Р}

1. Метса вээр Я. А., Векслер Н. Д., Сту лов А. С., Дифракция акустических импульсов на упругих телах, М., «Наука», 1979.

2. Векслер Н. Д., Рассеяние импульсов на упругих цилиндрах, Таллин, «Валгус», 1980.

3. Oberall, H., In: Physical Acoustics, ed. by W. P. Mason and R. N. Thurston, 10, Academic Press, New York, 1973, p. 1-60.

4. N e u b a u e r, W. G., In: Physical Acoustics, ed. by W. P. Mason and R. N. Thurston, 10, Academic Press, New York, 1973, p. 61-126.

5. В и к торов И. А., Акуст. ж., 25, № 1, 1-17 (1979).

6. Flax, L., N eubauer, W., J. Acoust. Soc. Amer., 61, № 2, 307-312 (1977).

7. G a u n a u r d, G. C., J. Acoust. Soc. Amer., 61, № 2, 360-368 (1977).

8. G a u n a urd, G. C., J. Acoust. Soc. Amer., 62, № 2, 503-512 (1977).

9. Векслер Н., Кор сун ски й В., Изв. АН ӘССР, Физ. Матем., 29, № 3, 296$306(1980)$.

10. Векслер Н., Корсунский В., Изв. АН ЭССР, Физ. Матем., 29, № 4, 387$397(1980)$.

Ннститут кибернетики

Академии наук Эстонской ССР

Поступила в редакцию

16/VI 1980

\section{N. VEKSLER, V. KORSUNSKI}

\section{AKUSTILISTE LAINETE MITTESTATSIONAARNE HAJUMINE ELASTSE TÄIDISEGA ELASTSETEL SILINDRITEL}

Artiklis on esitatud elastse täidisega elastse silindri poolt tekitatud akustilise lainevälja arvutamise tulemused. On vaadeldud vees asetsevat terassilindrit, mis on täidetud sulatatud räniga ning mille parameetrid määratakse valemiga (5). Lainevälja on jälgitud silindrist kaugel asuvas punktis $\left(r=10^{4}\right)$, kiirel $\Theta=0$. Rõhu sõltuvus sagedusest on esitatud joonisel 1. Väikeste sageduste piirkonnas on seda seost võrreldud analoogiliste, piirjuhtumite jaoks arvutatud seostega, mille puhul objektideks on akustiliselt jäik silinder, vedel koaksiaalse vedela täidisega silinder ja paksuseinaline elastne täidiseta silinder (joon. 2). Protsessi sõltuvus ajast on arvutatud valemiga (2) määratud langeva lainepaketi puhul, kui $x_{0}=9,84, \quad \delta_{0}=3 \tau_{0}$. Tabelis 1 on toodud hajunud lainevälja iseloomustavad suurused üksikute pakettide kaupa. On võrreldud faasi- ja grupikiirust nii ühe kui ka mitme erineva silindri puhul. Langeva lainepaketi sobiva valiku korral domineerib hajunud laineväljas peegeldunud lainepakett ja ühe elastse pinnalaine tekitatud pakett. Piirülesannetega õnnestub hästi modelleerida hajunud lainevälja üksikute komponentide tekkeprotsesse. Küllalt kõrge sageduse korral (kui $x_{0}=39$ ja $\delta_{0}=3 \tau_{0}$ ) võib eraldada peegeldunud ja hajunud lainepakette (joon. 5). On näidatud, et peegeldunud lainevälja rõhk avaldub kujul (7). On esitatud silindri raadiuse ja impedantsi määramise võimalus valemite (8) ja (9) abil. Täidise paksus ja impedants on määratud hajunud lainepaketi abil.

On esitatud ka muude silindri ja täidise materjale iseloomustavate suuruste leidmise metoodika. 


\section{N. VEKSLER, V. KORSUNSKI}

\section{TRANSIENT SCATTERING OF ACOUSTICAL WAVES ON ELASTIC CYLINDERS WITH AN ELASTIC FILLER}

Results of calculations of frequency- and time-depending acoustical pressure functions scattered by an elastic cylinder with an elastic filler are described in this paper. Calculations are performed in case of a steel cylinder with fused silica in water. The parameters of materials are given by the formulae (5). The observation point is situated in the far field: $r=10^{4}, \Theta=0$. In Fig. 1 we present a frequency-depending function. At low frequencies we compare it with the irequency-depending functions calculated for limit cases: acoustically rigid cylinder $(R)$, fluid cylinder with coaxial fluid filler $(F F)$, thick-walled elastic cylinder without any filler $(S E)$. This comparison is given in Fig. 2. The time-depending function was calculated for the initial wave pulse defined by the formulae (2) at $x_{0}=9.84$ and $\delta_{0}=3 \tau_{0}$. Parameters of reflected and re-radiated wave pulses are presented in Table 1. A comparison of phase and group velocities is made both for one scatterer and for different ones. It is demonstrated that in the case of wellchosen initial pulse in a time-depending function both reflected and re-radiated pulse dominate, caused by one elastic surface wave. Utilizing solutions of limit problems, we propose adequate models for processes of reflection, multiple reflection, and re-radiation. In the case of high frequency of the initial wave pulse $x_{0}=39, \delta_{0}=3 \tau_{0}$, pulses of multiple reflection and re-radiation are separated (see Fig. 5). It is shown that in a specular reflection domain the pressure can be written in the form (7).

We introduce a procedure of determining the radius and impedance of the cylinder by means of the formulae (8) and (9), the same as for thickness $H$ and impedance of the filler, using the multiple reflected wave pulse. We give an adaptive procedure of finding all the other parameters of materials of the cylinder and the filler. 